\title{
Association between Intraplaque Hemorrhage and Vascular Remodeling in Carotid Arteries: The Plaque at RISK (PARISK) Study
}

\author{
Kristine Dilba ${ }^{a, b} \quad$ Anouk C. van Dijk ${ }^{a, c}$ Geneviève A.J.C. Crombag ${ }^{d}$ \\ Anton F.W. van der Steen ${ }^{b}$ Mat J. Daemen ${ }^{\mathrm{e}}$ Peter J. Koudstaal $^{\mathrm{c}}$ \\ Paul J. Nederkoorn ${ }^{f}$ Jeroen Hendrikse ${ }^{g} \quad$ M. Eline Kooi ${ }^{d} \quad$ Aad van der Lugt ${ }^{a}$ \\ Jolanda J. Wentzel ${ }^{\mathrm{b}}$ \\ aRadiology and Nuclear Medicine, Erasmus MC, University Medical Center Rotterdam, Rotterdam, The Netherlands; \\ ${ }^{b}$ Cardiology, Erasmus MC, University Medical Center Rotterdam, Rotterdam, The Netherlands; ' Neurology, Erasmus

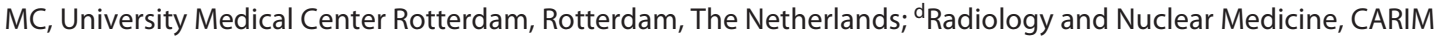 \\ School for Cardiovascular Diseases, Maastricht University Medical Center, Rotterdam, The Netherlands; ${ }^{\mathrm{e} A m s t e r d a m}$ \\ University Medical Center, University of Amsterdam, Pathology, Amsterdam, The Netherlands; ${ }^{\mathrm{f} N e u r o l o g y,}$ \\ University Medical Center Amsterdam, Amsterdam, The Netherlands; ${ }^{9}$ Radiology, University Medical Center Utrecht, \\ Utrecht, The Netherlands
}

\section{Keywords}

Carotid atherosclerosis · MRI · Plaque analysis · Vascular imaging $\cdot$ Vascular remodeling $\cdot$ Intraplaque hemorrhage

\footnotetext{
Abstract

Introduction: Vascular remodeling is a compensatory enlargement of the vessel wall in response to atherosclerotic plaque growth. We aimed to investigate the association between intraplaque hemorrhage (IPH), vascular remodeling, and luminal dimensions in recently symptomatic patients with mild to moderate carotid artery stenosis in which the differences in plaque size were taken into account. Materials and Methods: We assessed vessel dimensions on MRI of the symptomatic carotid artery in 164 patients from the Plaque At RISK study. This study included patients with recent ischemic neurological event and ipsilateral carotid artery stenosis $<70 \%$. The cross section with the largest wall
}

area (WA) in the internal carotid artery (ICA) was selected for analysis. On this cross section, the following parameters were determined: WA, total vessel area (TVA), and lumen area (LA). Vascular remodeling was quantified as the remodeling ratio (RR) and was calculated as TVA at this position divided by the TVA in an unaffected distal portion of the ipsilateral ICA. Adjustment for WA was performed to correct for plaque size. Results: Plaques with IPH had a larger WA $\left(0.56\right.$ vs. $\left.0.46 \mathrm{~cm}^{2} ; p<0.001\right)$, a smaller LA ( 0.17 vs. $0.22 \mathrm{~cm}^{2}$; $p=0.03$ ), and a higher RR ( 2.0 vs. $1.9 ; p=0.03$ ) than plaques without IPH. After adjustment for WA, plaques containing IPH had a smaller $\mathrm{LA}(B=-0.052, p=0.01)$ than plaques without IPH, but the RR was not different. Conclusion: After correcting for plaque size, plaques containing IPH had a smaller LA than plaques without IPH. However, RR was not different.

(C) 2020 The Author(s)

Published by S. Karger AG, Basel karger@karger.com www.karger.com/ced

Karger $\stackrel{2}{\%}$

BOPEN ACCESS (c) 2020 The Author(s)

Published by S. Karger AG, Basel

This is an Open Access article licensed under the Creative Commons Attribution-NonCommercial-4.0 International License (CC BY-NC) (http://www.karger.com/Services/OpenAccessLicense), applicable to the online version of the article only. Usage and distribution for commercial purposes requires written permission.
Jolanda J. Wentzel

Cardiology, Erasmus MC, University Medical Center Rotterdam

Dr. Molewaterplein 40

NL-3015 Rotterdam (The Netherlands)

j.wentzel@erasmusmc.nl 


\section{Introduction}

Atherosclerotic disease of the extracranial carotid arteries is an important cause of ischemic stroke [1]. The risk of recurrent stroke increases with degree of stenosis in the carotid artery [2].

Rupture-prone plaques are characterized by a large lipidrich necrotic core (LRNC) covered by a thin fibrous cap [3], the presence of intraplaque hemorrhage (IPH) [4-6] and positive vascular remodeling [7], which can be noninvasively assessed and measured with MR imaging [8]. IPH is caused by a bleeding within the atherosclerotic plaque which may originate from small leaky and fragile neovessels present in the plaque or alternatively from fissuring or rupturing of the fibrous cap [9-11]. Positive vascular remodeling is a gradual compensatory enlargement of the vessel wall that initially prevents lumen narrowing in response to atherosclerotic plaque growth [12]. As a result, lumen size does not reflect the extent of atherosclerotic plaques [13]. At later stages of the disease, remodeling is still present but not fully effective to prevent lumen narrowing [12]. In particular, carotid plaques that contain IPH showed lumen narrowing [14-16]. It is unclear why carotid plaques with IPH lead to lumen narrowing and which role vascular remodeling plays in this process. To date there are only a few studies that investigated the association between IPH and vascular remodeling in carotid arteries $[7,17,18]$. However, previous studies did not take into account that IPH occurs more frequently in larger plaques. Thereby the comparison of vascular remodeling between plaques with and without IPH might be biased because that may only reflect a comparison between smaller and larger plaques. The aim of this cross-sectional study was to investigate the association between IPH, vascular remodeling, and luminal dimensions in recently symptomatic patients with mild to moderate carotid artery stenosis with MRI in which we take the differences in plaque size into account.

\section{Materials and Methods}

\section{Study Population}

This study is embedded within the Plaque At RISK (PARISK) study. From September 2010 till December 2014, a total of 240 patients were included with recent $(<3$ month) transient ischemic attack, amaurosis fugax, or minor stroke due to ischemia in the territory of the carotid artery and a 30-69\% ipsilateral carotid artery stenosis. Details of the study design and cardiovascular risk factors have been previously described [19].

The study protocol was approved by the institutional medical Ethics Committees and conforms to the ethical guidelines of the 1975 Declaration of Helsinki. Written informed consent was obtained from each participant before enrollment.

Intraplaque Hemorrhage and Vascular Remodeling
MR Imaging Data Acquisition and Analysis

All examinations were performed on $3.0 \mathrm{~T}$ whole body MRI scanners. Imaging protocols included 5 sequences that were comparable between centers (Philips: 3D time-of-flight fast field echo, 3D T1-weighted inversion recovery turbo field echo, 2D T2weighted turbo spin echo, 2D T1-weighted quadruple inversion recovery turbo spin echo pre- and post-contrast; GE: 3D fast spoiled gradient echo, 3D T1-weighted spoiled gradient echo, 2D T2-weighted double inversion recovery fast spin echo, 2D T1weighted double inversion recover fast spin echo pre- and postcontrast). A more detailed description of the sequences is provided in the study design article [19].

Six observers, who were trained in the same institution to delineate plaque components, evaluated the MR images of the symptomatic carotid artery with the VesselMass software (Department Radiology, Leiden University Medical center, The Netherlands). Furthermore, observers were blinded to clinical data and other imaging tests. There were in average 15 transverse adjacent cross sections of $2 \mathrm{~mm}$ each covering the entire plaque. MR images of different sequences were registered after delineating the lumen and outer vessel wall. Regions with a wall thickness $>2 \mathrm{~mm}$ was defined as plaque [20]. Plaque components (LRNC, calcifications, and IPH) were manually segmented using a standardized protocol as described in the study design article [19]. In short, IPH was defined as hyperintense signal in the bulk of the plaque compared with the adjacent sternocleidomastoid muscle in the 3D T1-weighted inversion recovery turbo field echo or 3D T1-weighted spoiled gradient echo images [21]. Good sensitivity, specificity, and interobserver agreement were reported for the detection of IPH in the studies that used sequences like ours $[22,23]$. The LRNC is delineated as an isointense to hyperintense region within the bulk of the plaque on pre-contrast T1w MRI that does not enhance on the post-contrast T1w MRI. In addition, IPH volume was always considered as part of the LRNC [22]. Fibrous cap (FC) status was divided in 2 categories: thick versus thin-or-ruptured (TRFC) based on previously published criteria [24]. Three cross sections were selected for quantitative analysis: the cross section with the maximal wall area (WA) in the symptomatic internal carotid artery (ICA) and 2 cross sections adjacent to each other without plaque and located distally to the previous mentioned cross section. The lumen area (LA) and the WA were calculated based on the lumen and wall contours using the VesselMass software and the following parameters were derived. Total vessel area (TVA) was calculated as LA + WA (Fig. 1). Reference TVA (TVA REF ) was the average TVA in the 2 distal cross sections without plaque. Plaque burden was $\mathrm{WA} / \mathrm{TVA} \times 100 \%$. The maximal vessel wall thickness was determined as the greatest wall thickness within the cross section. Remodeling ratio (RR) was calculated as TVA/TVA $A_{\text {REF. }}$ In addition, the number of cross sections between the bifurcation and the (a) cross section with maximal WA and (b) reference cross sections were recorded.

\section{Statistical Analysis}

Categorical variables are presented as absolute numbers and relative frequencies. Continuous variables are presented as mean \pm SD or as median (interquartile range). $T$ test was used to compare continues variables. Ln-transformation was performed to achieve a normal distribution. Mann-Whitney U Test was used to compare non-normal distributed continuous variables. Categorical data were evaluated using $\chi^{2}$ test. 


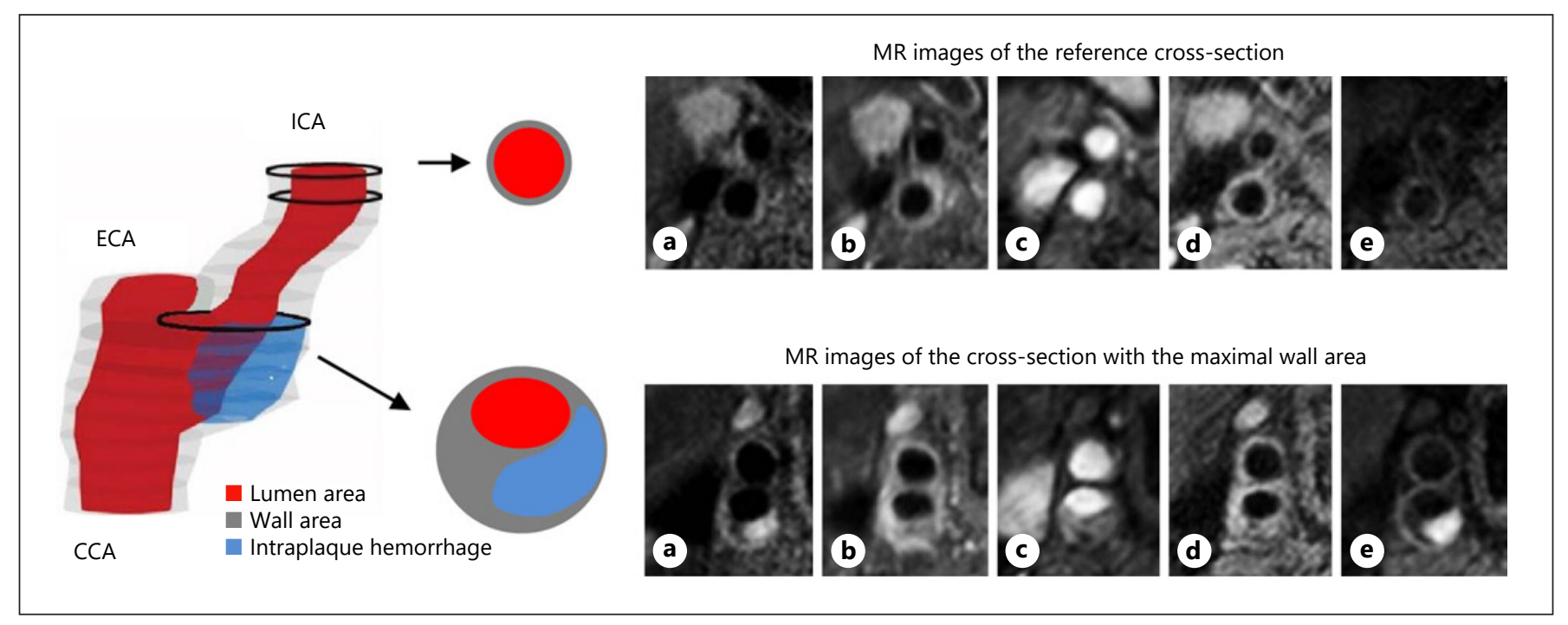

Fig. 1. Three-dimensional representation of a carotid artery with IPH. On the top row: axial MRI of the reference cross section localized in non-atherosclerotic distal portion of the ICA. On the lower row: axial MRI of the cross section selected for analysis localized at the point of the maximal WA in ICA. 2D T1w-QIR-TSE pre-

The association between IPH and geometrical parameters was evaluated using linear regression analysis with geometrical parameters (WA, maximal wall thickness, TVA, RR, LA, and plaque burden) as outcome, and IPH (present, absent) as input variable (univariable analysis) and adjusted for WA (multivariable analysis), and additionally adjusted for age and sex. We adjusted the analysis for WA because both vascular remodeling and IPH are more prevalent in vessels with advanced atherosclerosis. This adjustment allows the comparison of IPH and non-IPH plaques with the same WA.

Because IPH was not always present in the selected cross section with maximal WA despite that IPH was present in the plaque, analysis was repeated by comparing those patients with IPH present in the selected cross section versus no IPH present.

To investigate whether other plaque components influenced the geometrical parameters as well, we repeated the above described analysis for LRNC (present, absent) and TRFC (present, absent). Furthermore, since in the plaque LRNC can be present simultaneously with IPH, we analyzed LRNC (present, absent) in plaques without IPH, to study the influence of this plaque component on geometrical parameters independent from IPH.

A value of $p<0.05$ was considered significant ( 2 sided). All calculations were performed using SPSS version 21 (IBM Corp, 2012).

\section{Results}

\section{Patients Characteristics}

From 240 included patients, 76 patients were not included in the current analyses because MRI was not performed $(n=11)$, had poor image quality $(n=10)$, absence contrast (a); 2D T1w-QIR-TSE post-contrast (b); 3D TOF-FFE (c); 2D T2w TSE (d); 3D T1w-IR-TFE (e). IPH, intraplaque hemorrhage; WA, wall area; LA, lumen area; ICA, internal carotid artery; ECA, external carotid artery; CCA, common carotid artery.

Table 1. Baseline clinical characteristics of the patients

\begin{tabular}{lllc}
\hline & $\begin{array}{l}\text { IPH absent } \\
n=107\end{array}$ & $\begin{array}{l}\text { IPH present } \\
n=57\end{array}$ & $p$ value \\
& $60(55 \%)$ & $49(86 \%)$ & $<0.001$ \\
\hline $\begin{array}{l}\text { Sex (male) } \\
\text { Age }\end{array}$ & $67 \pm 4$ & $71 \pm 7$ & 0.02 \\
Classification event, $n(\%)$ & & & \\
$\quad$ TIA & $56(52)$ & $20(35)$ & 0.07 \\
$\quad$ Amaurosis fugax & $12(11)$ & $6(11)$ & \\
$\quad$ Stroke & $39(36)$ & $31(54)$ & \\
BMI, kg/m & $27 \pm 4$ & $26 \pm 4$ & 0.11 \\
Hypertension, $n(\%)$ & $70(66)$ & $39(70)$ & 0.64 \\
Hypercholesterolemia, $n(\%)$ & $52(53)$ & $34(63)$ & 0.38 \\
Diabetes mellitus, $n(\%)$ & $26(25)$ & $13(23)$ & 0.85 \\
Smoking status, $n(\%)$ & & & \\
$\quad$ Never & $24(23)$ & $9(17)$ & 0.10 \\
$\quad$ Former & $50(47)$ & $35(65)$ & \\
$\quad$ Current & $32(30)$ & $10(18)$ & \\
\hline
\end{tabular}

IPH, intraplaque hemorrhage; TIA, transient ischemic attack.

of reference segment $(n=43)$ or presence of plaque in the bulb instead of the ICA $(n=12)$. Finally, 164 patients were included in the current analyses. In 57 of the patients (35\%), IPH was present in the plaque. Patients with IPH were more frequently male ( $86 \%$ vs. $55 \% ; p<0.001)$ and were older (71 vs.67; $p=0.02$ ) compared to patients without IPH (Table 1).
96

Cerebrovasc Dis 2021;50:94-99

DOI: $10.1159 / 000511935$
Dilba et al. 
Table 2. Comparison of geometric parameters between plaques with IPH and without IPH

\begin{tabular}{|c|c|c|c|c|c|c|}
\hline & $\begin{array}{l}\text { IPH absent } \\
n=107\end{array}$ & $\begin{array}{l}\text { IPH present } \\
n=57\end{array}$ & $\begin{array}{l}\text { Univariable analysis }^{1} \\
(\mathrm{~B} ; 95 \% \mathrm{CI})\end{array}$ & $p$ value & $\begin{array}{l}\text { Multivariable analysis }{ }^{2} \\
\text { (B; } 95 \% \mathrm{CI} \text { ) }\end{array}$ & $p$ value \\
\hline $\mathrm{WA}, \mathrm{cm}^{2}$ & $0.46(0.39-0.55)$ & $0.56(0.45-0.71)$ & $0.132(0.079 ; 0.186)$ & $<0.001$ & $\mathrm{na}^{3}$ & na \\
\hline Total vessel area, $\mathrm{cm}^{2}$ & $0.7(0.59-0.81)$ & $0.76(0.62-0.94)$ & $0.090(0.021 ; 0.159)$ & 0.01 & $-0.052(-0.093 ;-0.011)$ & 0.01 \\
\hline Plaque burden, \% & $66(60-74)$ & $74(67-84)$ & $7.873(4.469-11.277)$ & $<0.001$ & $4.298(0.984 ; 7.612)$ & 0.01 \\
\hline $\mathrm{LA}, \mathrm{cm}^{2}$ & $0.22(0.16-0.31)$ & $0.17(0.13-0.24)$ & $-0.042(-0.081 ;-0.004)$ & 0.03 & $-0.052(-0.093 ;-0.01)$ & 0.01 \\
\hline
\end{tabular}

WA, wall area; LA, lumen area; RR, remodelling ratio; IPH: intraplaque hemorrhage. ${ }^{1}$ Univeriable analysis: IPH presence. ${ }^{2}$ Multivariable analysis: IPH presence and wall area. ${ }^{3}$ Not applicable.

Comparison between Plaques with and without IPH

Atherosclerotic plaques with IPH had larger WA than plaques without IPH (WA 0.56 vs. $0.46 \mathrm{~cm}^{2} ; p<0.001$ ) and a larger maximal wall thickness ( 4.1 vs. $3.3 \mathrm{~mm}$; $p<$ $0.001)$. In plaques with IPH the TVA was larger $(0.76$ vs. $\left.0.70 \mathrm{~cm}^{2} ; p=0.01\right)$ as well as the RR (2.0 vs. $1.9 ; p=$ $0.03)$. This resulted in a smaller LA $\left(0.17\right.$ vs. $0.22 \mathrm{~cm}^{2} ; p=$ 0.03 ) and a higher plaque burden (74 vs. $66 \% ; p<0.001)$ in plaques with IPH compared to plaques that did not contain IPH (Table 2). The reference TVA had the same size $\left(0.37[0.33-0.43] \mathrm{cm}^{2}\right.$ vs. $0.36[0.32-0.42] \mathrm{cm}^{2} ; p=$ $0.5)$ and reference cross sections were equally far located from the bifurcation (7.5 [6.5-8.7] vs. $7.5[6.5-7.5] ; p=$ $0.19)$. Moreover, the location of the cross section with maximal WA was not different for plaques with and without IPH (1 [1-2] vs.1 [1-2]; $p=0.31)$.

After adjustment for WA, plaques with IPH showed a smaller TVA $(B=-0.052 ; p=0.01)$ and LA $(B=-0.052$; $p=0.01)$. However, after adjustment for WA, there was no significant difference in RR (Table 2). Additional adjustment for sex and age did not change the results for all parameters.

IPH was localized within the selected slice of maximal WA in 40 (70\%) of the cases. When we repeated the analysis for only the plaques that contained IPH within the slice with maximal WA $(n=40)$ versus plaques without IPH at all, similar results were found for the univariable and multivariable analysis.

\section{Comparison between Plaques with and without Other Plaque Components}

When geometrical parameters were compared for plaques with LRNC versus non-LRNC, similar to IPH, after adjustment for WA, TVA $(B=-0.044, p=0.026)$ and LA $(B=-0.044$. $p=0.026)$ were significantly smaller but RR was not different $(-0.081, p=0.274)$, (see online

Intraplaque Hemorrhage and Vascular Remodeling suppl. Table 1; for all online suppl. material, see www. karger.com/doi/10.1159/000511935). For plaques with TRFC versus non-TRFC, similar results were obtained (online suppl. Table 2).

To investigate the relationship between LRNC and geometrical parameters independent from IPH, subgroup analysis on all plaques without IPH $(n=107)$ was repeated and demonstrated that plaques that contain LRNC $(n=43)$ do not differ in LA, TVA, and RR from plaques without LRNC $(n=64)$ (online suppl. Table 3 ).

\section{Discussion}

This study demonstrates that plaques with IPH had a larger plaque area and burden, were thicker, and had a smaller LA with a higher RR compared to plaques without IPH. Furthermore, after adjustment for WA, plaques with IPH had a smaller TVA, resulting also in a smaller LA but did not differ in RR compared to the non-IPH plaques.

IPH seems to be a natural process of plaque development that occurs in more advanced stages of atherosclerosis. Increase in atherosclerotic disease results in an increase in plaque area and thereby vascular remodeling also increases. Therefore, comparing vascular remodeling in plaques with and without IPH without adjustment for plaque size would not be an appropriate approach. After adjustment of the remodeling measures (TVA and RR) for plaque size, represented by the WA, we demonstrated that plaques with IPH had a smaller TVA and LA. However, adjustment for WA did not result in a smaller RR. We have 2 explanations for this inconsistency. First, it cannot be excluded that the size of the carotid bulb before plaque formation was different among the IPH and the non-IPH group. Second, in crosssectional studies, vascular remodeling is typically measured as the ratio between TVA at the studied plaque site and 
TVA at a non-diseased ipsilateral part of the vessel (reference) $[7,17,18]$. In general, it is assumed that the reference site gives information about the dimension of the vessel before plaque growth. However, in the non-atherosclerotic carotid arteries, only a moderate correlation was observed between the carotid bulb size and the distal ipsilateral unaffected ICA [25]. Thus, the TVA of the reference site probably does not give accurate information on the initial size of carotid artery before plaque growth. Therefore, the use of the distal reference cross section and thus the RR as a measure for vascular remodeling in carotid arteries can be questioned. However, alternatives are scarce in a cross-sectional study design. Future longitudinal studies can answer the question why plaques with IPH lead to lumen narrowing and which role vascular remodeling plays in this process. It might be hypothesized that due to accelerated plaque growth because of IPH, vascular remodeling is not fast enough to keep the vessel lumen its original dimensions. Thereby, lumen narrowing occurs.

The analysis was performed in the ICA cross section with the maximal WA in contrast to others who use the cross section of maximal stenosis $[7,17,18,26]$ and an average TVA to calculate RR [18]. First, we expected to find IPH in the cross section with the largest WA. Indeed our data show that in $70 \%$ of the cases IPH was located in the cross section with the largest WA. Second, our patients had a gradual lumen decrease; therefore, the cross section with the smallest LA was often located in the most distal part of the ICA, which was not always diseased.

Our results are difficult to compare with previous studies in which adjustment for plaque size has not been performed and other definitions of remodeling were used. We can only compare previous results to our unadjusted results. Similar to Fukuda et al. [17], we found that plaques containing IPH were associated with a higher RR. In contrast to our study, Saam et al. [18] used the average TVA compared to a reference site to define the RR. However, we are convinced that it is important to use only 1 cross section with maximal vascular remodeling, that is expected to be at the cross section with maximal WA. Averaging may lead to weakening of existing correlations between parameters. For example, Saam et al. [18] did not observe a difference in vascular RR, based on average RR, among plaques with different American Heart Association classification subtypes of which lesion type VI contains IPH. However, when analysis was repeated using only 1 single cross section at maximal stenosis, differences between lesion type VI and VII appeared significant.

Our study has several limitations. First, the study is a cross-sectional study, which limits us to draw firm conclu- sions about the causal role of IPH in vascular remodeling and lumen narrowing. Second, we analyzed only 1 cross section selected from the plaque with maximal WA, which not always contained IPH, even though IPH was present in the plaque. However, when the analysis was performed on the cross sections with maximal WA containing also IPH, the same results were found. So, the cross section with maximal WA represents the global response of the plaque. Third, several plaque components are often simultaneously present in the plaque. Therefore, to study their independent influence on geometrical parameters is challenging. If plaques were stratified according to LRNC or TRFC similar to IPH, a smaller lumen and total vessel area were observed without differences in RR (online suppl. Tables 1,2). The main explanation for this observation is the high correlation/colocalization of plaque components. If plaques were stratified according to the presence of LRNC or TRFC, 57\% of the cases and $76 \%$ of the cases respectively also contained IPH in the plaque. To answer the question whether the observed differences in LA, TVA, and absence of difference in RR for LRNC were fully dominated by IPH, a subgroup analysis was performed for plaques without IPH to study the relationship between LRNC and the geometrical parameters. Interestingly, comparing plaques with and without LRNC but without IPH, the observed differences in LA and TVA were not present anymore, implying that LRNC by itself is not involved in vascular remodeling and lumen narrowing. For TRFC, no subgroup analysis was performed since according to protocol the FC is the area between LRNC and/or IPH and lumen and therefore is always linked to either LRNC or IPH.

\section{Conclusion}

After adjustment for plaque size, plaques containing IPH have a smaller LA than the plaques without IPH. However, the vascular remodeling was not different.

\section{Statement of Ethics}

The study was approved by the institutional medical Ethics Committee of all participating centers and conforms to the ethical guidelines of the 1975 Declaration of Helsinki. Written informed consent was obtained from each participant before enrollment.

\section{Conflict of Interest Statement}

The authors declare no conflict of interest.
98

Cerebrovasc Dis 2021;50:94-99 DOI: $10.1159 / 000511935$
Dilba et al. 


\section{Funding Sources}

This research was performed within the framework of the Center for Translational Molecular Medicine (www.ctmm.nl), project PARISK (Plaque At RISK; Grant 01C-202) and supported by the Dutch Heart Foundation. Kristina Dilba was in part supported by STW project number 10813.

\section{Authors Contributions}

K.D., J.J.W., A.v.d.L. were involved in the concept, design, and execution of the study, in the interpretation of the data, and in writing and critically reviewing the manuscript. A.C.v.D., G.A.J.C.C., A.F.W.v.d.S., M.J.A.P.D., P.J.K., P.J.N., J.H., and M.E.K. were involved in the interpretation of the data and in critically reviewing the manuscript. All authors approved the final version to be submitted for publication.

\section{References}

1 Petty GW, Brown RD Jr, Whisnant JP, Sicks JD, O'Fallon WM, Wiebers DO. Ischemic stroke subtypes: a population-based study of incidence and risk factors. Stroke. 1999; 30(12):2513-6.

2 Randomised trial of endarterectomy for recently symptomatic carotid stenosis: final results of the mrc european carotid surgery trial (ecst). Lancet 1998;351(9113):1379-87.

3 Yuan C, Zhang SX, Polissar NL, Echelard D, Ortiz G, Davis JW, et al. Identification of fibrous cap rupture with magnetic resonance imaging is highly associated with recent transient ischemic attack or stroke. Circulation. 2002;105(2):181-5

4 Altaf N, MacSweeney ST, Gladman J, Auer DP. Carotid intraplaque hemorrhage predicts recurrent symptoms in patients with highgrade carotid stenosis. Stroke. 2007;38(5) $1633-5$.

5 Altaf N, Daniels L, Morgan PS, Auer D, MacSweeney ST, Moody AR, et al. Detection of intraplaque hemorrhage by magnetic resonance imaging in symptomatic patients with mild to moderate carotid stenosis predicts recurrent neurological events. J Vasc Surg. 2008;47(2):337-42.

6 Schindler A, Schinner R, Altaf N, Hosseini AA, Simpson RJ, Esposito-Bauer L, et al. Prediction of stroke risk by detection of hemorrhage in carotid plaques: meta-analysis of individual patient data. JACC Cardiovasc Imaging. $2020 \mathrm{Feb} ; 13(2 \mathrm{Pt}$ 1):395-406.

7 Miura T, Matsukawa N, Sakurai K, Katano H, Ueki Y, Okita K, et al. Plaque vulnerability in internal carotid arteries with positive remodeling. Cerebrovasc Dis Extra. 2011;1(1):5465.

8 Yuan C, Kerwin WS, Yarnykh VL, Cai J, Saam $\mathrm{T}$, Chu B, et al. Mri of atherosclerosis in clinical trials. NMR Biomed. 2006;19(6):636-54.

9 Virmani R, Kolodgie FD, Burke AP, Finn AV, Gold HK, Tulenko TN, et al. Atherosclerotic plaque progression and vulnerability to rupture: angiogenesis as a source of intraplaque hemorrhage. Arterioscler Thromb Vasc Biol. 2005;25(10):2054-61.
10 Crombag GAJC, Schreuder FHBM, van Hoof RHM, Truijman MTB, Wijnen NJA, Vöö SA, et al. Microvasculature and intraplaque hemorrhage in atherosclerotic carotid lesions: a cardiovascular magnetic resonance imaging study. J Cardiovasc Magn Reson. 2019;21(1): 15.

11 Daemen MJ, Ferguson MS, Gijsen FJ, Hippe DS, Kooi ME, Demarco K, et al. Carotid plaque fissure: an underestimated source of intraplaque hemorrhage. Atherosclerosis. 2016;254:102-8.

12 Glagov S, Weisenberg E, Zarins CK, Stankunavicius R, Kolettis GJ. Compensatory enlargement of human atherosclerotic coronary arteries. N Engl J Med. 1987;316(22):1371-5.

13 Zhao H, Zhao X, Liu X, Cao Y, Hippe DS, Sun $\mathrm{J}$, et al. Association of carotid atherosclerotic plaque features with acute ischemic stroke: a magnetic resonance imaging study. Eur J Radiol. 2013;82(9):e465-70.

14 Takaya N, Yuan C, Chu B, Saam T, Polissar NL, Jarvik GP, et al. Presence of intraplaque hemorrhage stimulates progression of carotid atherosclerotic plaques: a high-resolution magnetic resonance imaging study. Circulation. 2005;111(21):2768-75.

15 Sun J, Underhill HR, Hippe DS, Xue Y, Yuan C, Hatsukami TS. Sustained acceleration in carotid atherosclerotic plaque progression with intraplaque hemorrhage: a long-term time course study. JACC Cardiovasc Imaging. 2012;5(8):798-804.

16 Underhill HR, Yuan C, Yarnykh VL, Chu B, Oikawa M, Polissar NL, et al. Arterial remodeling in [corrected] subclinical carotid artery disease. JACC Cardiovasc Imaging. 2009; 2(12):1381-9.

17 Fukuda K, Iihara K, Maruyama D, Yamada N, Ishibashi-Ueda H. Relationship between carotid artery remodeling and plaque vulnerability with t1-weighted magnetic resonance imaging. J Stroke Cerebrovasc Dis. 2014; 23(6): 1462-70

18 Saam T, Habs M, Buchholz M, Schindler A, Bayer-Karpinska A, Cyran CC, et al. Expansive arterial remodeling of the carotid arteries and its effect on atherosclerotic plaque composition and vulnerability: an in-vivo blackblood $3 \mathrm{t} \mathrm{cmr}$ study in symptomatic stroke patients. J Cardiovasc Magn Reson. 2016;18:11.
19 Truijman MT, Kooi ME, van Dijk AC, de Rotte AA, van der Kolk AG, Liem MI, et al. Plaque at risk (parisk): prospective multicenter study to improve diagnosis of high-risk carotid plaques. Int J Stroke. 2014;9(6):74754.

20 Selwaness M, van den Bouwhuijsen Q, Mattace-Raso FU, Verwoert GC, Hofman A, Franco $\mathrm{OH}$, et al. Arterial stiffness is associated with carotid intraplaque hemorrhage in the general population: the rotterdam study. Arterioscler Thromb Vasc Biol. 2014;34(4): 927-32.

21 Yuan C, Mitsumori LM, Ferguson MS, Polissar NL, Echelard D, Ortiz G, et al. In vivo accuracy of multispectral magnetic resonance imaging for identifying lipid-rich necrotic cores and intraplaque hemorrhage in advanced human carotid plaques. Circulation. 2001;104(17):2051-6.

22 Cappendijk VC, Cleutjens KB, Heeneman S, Schurink GW, Welten RJ, Kessels AG, et al. In vivo detection of hemorrhage in human atherosclerotic plaques with magnetic resonance imaging. J Magn Reson Imaging. 2004;20(1): 105-10.

23 Bitar R, Moody AR, Leung G, Symons S, Crisp $\mathrm{S}$, Butany J, et al. In vivo $3 \mathrm{~d}$ high-spatial-resolution mr imaging of intraplaque hemorrhage. Radiology. 2008;249(1):259-67.

24 Kwee RM, van Engelshoven JM, Mess WH, ter Berg JW, Schreuder FH, Franke CL, et al. Reproducibility of fibrous cap status assessment of carotid artery plaques by contrastenhanced mri. Stroke. 2009;40(9):3017-21.

25 Williams MA, Nicolaides AN. Predicting the normal dimensions of the internal and external carotid arteries from the diameter of the common carotid. Eur J Vasc Surg. 1987;1(2): 91-6.

26 Hardie AD, Kramer CM, Raghavan P, Baskurt E, Nandalur KR. The impact of expansive arterial remodeling on clinical presentation in carotid artery disease: a multidetector ct angiography study. AJNR Am J Neuroradiol. 2007;28(6):1067-70. 